\title{
Assessing quality control: Evaluating the quality audit ${ }^{1}$
}

\author{
Justin D. Nguyen* and Carma R. Hogue \\ U.S Census Bureau, Washington D.C., NY, USA
}

\begin{abstract}
Effective quality control systems are the foundation for successful manufacturers and data publishers. For years, statisticians have proposed theories and findings to improve data product quality. The Economic Directorate of the U.S. Census Bureau collects various economic data with a requirement to accurately capture and analyze our data and conduct quality audits to ensure programs correctly identify problems to save time and money and ensure quality. Our primary objective is to assess our program areas' compliance with best practices, particularly in the area of dissemination, to ensure that statistically sound practices are used in the collection of data and in the presentation of results to the public. These data have gone through rigorous quality control procedures to assure the highest possible quality and consistency. In this paper, we discuss the first two rounds of quality audit processes that i) identified inspection goals and inspection plans, ii) detected data quality, iii) communicated quality expectations and provided recommendations based on the audit results. In the remainder of the paper we discuss measures to correct system challenges and introduce quality assurance measures throughout the survey life cycle.
\end{abstract}

Keywords: U.S Census Bureau, quality control, integrated project processes

\section{Introduction}

The U.S. Census Bureau's Economic Directorate is responsible for producing 1) key monthly and quarterly indicators of the state of the U.S. economy, 2) the Economic Census that yields the U.S. government's official 5-year measure of the nation's business and economy, and 3) various other intercensal surveys that feature industry and geographic statistics of selected economic sectors that aid in a deeper understanding of the nation's economic health. Ten years ago, the Economic Directorate committed to establishing a formal quality management program to ensure that all surveys met the quality guidelines formally released by the Office of Management and Budget (OMB) in 2006 as well as the Census Bureau standards most recently reissued in 2013. The Quality Audit Staff developed an audit structure to review surveys every five years, regularly

\footnotetext{
${ }^{1}$ This paper was presented at the Q2016 conference.

${ }^{*}$ Corresponding author: Justin D. Nguyen, U.S Census Bureau, 4600 Silver Hill Road, Washington D.C., 20233-1912, NY, USA. E-mail: Justin.D.Nguyen@Census.gov.
}

reported the audit findings to senior leadership, and monitored progress towards standards compliance for each of the surveys.

After a decade of audits, most programs and surveys have been through the audit twice. In examining the ten years of findings, we can see that quality of most surveys has improved, but there are some standards that almost every survey has failed, sometimes because the process or system that the survey is using is not compliant with a particular standard. For example, improper calculation of response rates can occur because of a lack of appropriate edit and imputation flags. Sometimes, the audit failure has been because a nonresponse bias study was not conducted or a quality assurance checklist has not been in place. A lack of edit evaluation studies has led to over-editing of data and an inefficient use of human resources. We also looked at erroneous dissemination reports over the time period to determine what errors led to the release of erroneous data products. A number of findings have been consistent enough to warrant a temporary cessation of the quality audits and a redirection of the Quality Audit Staff to insure that procedural improvements are made. 
As new enterprise systems are built, the Quality Audit Staff will ensure that reports, flags, quality assurance measures, and checks are added into the systems to constantly monitor procedures.

In this paper, we will give a brief overview of the current auditing process in Section 2. General quality assurance measures that will be put in place are covered in Section 3. Section 4 covers the plan for the integration of quality control activities into our processes. Section 5 will look to the future and examine those decisions that must be made prior to the next cycle of audits.

\section{Overview of the current auditing process}

The Quality Assurance Program's audit process in the Economic Directorate consists of four main elements. The first element is the pre-audit activities. In the pre-audit activities, the Lead Auditor and the Assistant Lead Auditor plan for all audit activities and conduct training for staff involved in the audit program. The internal audit comprises the second element with the audited program's staff members reviewing their practices and procedures documentation and completing a formal checklist that checks compliance with OMB's guidelines and the Census Bureau's quality standards for all seven phases of a survey's life cycle. These phases are:

- Survey Development

- Collection of Data

- Processing and Editing of Data

- Production of Estimates and Projections

- Data Analysis

- Review Procedures

- Dissemination of Information Products

The checklist is divided into three main parts. The first part consists of the instructions for the audit process. The second part provides examples of auditor citations in the OMB-Census checklist. The final part of the checklist provides a detailed OMB-Census standards checklist. Once the checklist has been completed by the survey staff, an independent quality audit team that usually consists of the Lead Auditor, Assistant Lead Auditor and a volunteer auditor performs an external audit. After the external audit is completed, the program area is required to develop an action plan to address how they propose to accomplish the recommendations made by the external quality audit team on their final report. The complete refined audit process is shown in Fig. 1 below.

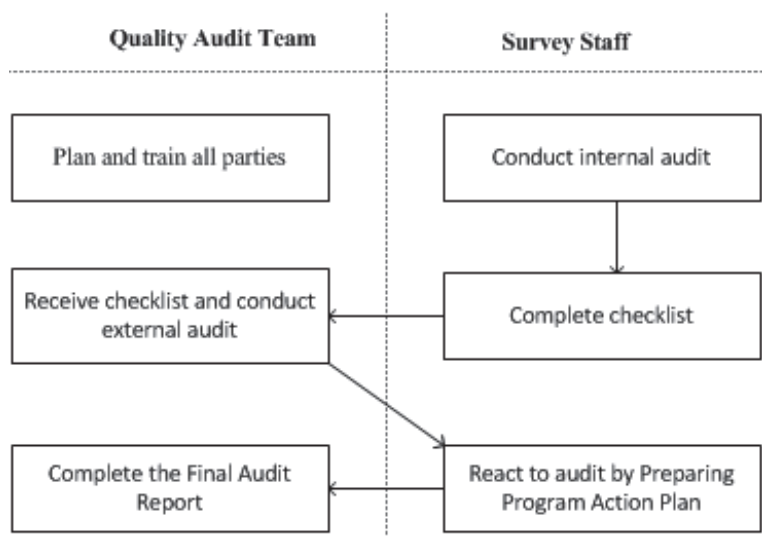

Fig. 1. The Refined Audit Diagram Process.

The quality of an individual audit depends upon the input provided by each program area. Detailed consideration needs to be given to the nature and extent of audit evidence obtained to determine if the supplied materials misstate actual procedures, the appropriateness of the relevant audit judgements made, and to compliance with relevant standards. Because the resources available for conducting the external audits are limited, the Quality Audit Staff asks each survey staff to provide us as much evidence as possible to support that their work has been done correctly in accordance to OMB and Census Bureau standards.

During the course of these auditing processes, the auditors routinely discovered practices and procedures that could be improved upon. Auditors often spotted systematic problems within the same entity for multiple audit cycles, as well as systematic problems across surveys. The first common issue was missing reports to support that a nonresponse bias analysis had been conducted for those surveys that suggested the potential for bias to occur. The second common issue was a lack of proper survey documentation. For example, during the first five-year auditing cycle, survey documentation was noncompliant or recommended for improvement in a little under one-third of all audit programs [5]. The Economic Directorate's audit process will be considered in this instance as a facilitator of tangible evidence, helping to validate the argument that improper document creation and storage negatively affected the Economic Directorate's primary objective of creating the highest quality statistics possible. These issues led to a suggestion that we must include a plan for the integration of quality control activities into our processes. This new quality control system will archive all activities in a survey's lifecycle. The details of this quality control process are discussed in Section 4. 


\section{General quality control and quality assurance}

In general, all surveys and data collection activities conducted by the Economic Directorate that result in an external publication must be included within the scope of the quality audit program and quality assurance procedures. However, the adoption of quality assurance practices is still not as strong as it could be. One main problem is that the usage of these quality assurance procedures is often not clear, especially, when and how these quality procedures are to be used in an existing process. Regardless of the amount of time and effort, each program area has put into quality assurance during the last decade of auditing, the same audit failure has been discovered multiple times within the same survey and across surveys. Thus, we must develop more detailed quality assurance procedures into the enterprise systems. These quality assurance procedures will serve as ongoing processes required for all surveys and data collection activities conducted by the Economic Directorate. Each activity needs to be performed in accordance with $\mathrm{OMB}$ and Census $\mathrm{Bu}$ reau auditing standards and are subject to the Economic Directorate's quality control processes. For all survey activities, a quality control system must be implemented to ensure the accuracy and completeness of the program plans, including all schedules, cost estimates, agreements, statements of work, survey design, sampling design, estimation methodologies, etc. These quality control procedures will monitor processes and take remedial action, as appropriate. Since the audit involves difficult decisions and judgments made by staff at different experience levels, and sometimes under tight time constraints, a necessary requirement is that the survey staff must develop a comprehensive internal audit plan or checklist that is relatively easy for the Quality Audit Staff to follow. The survey staff must also provide a checklist that is exceptionally well organized, and all activities must be well documented. The survey manager must enlist the expertise of all methodological and analytical staff members who might be able to provide specific organizational insight to the internal audit. After the survey staff obtain the checklist, quality assurance checks must be performed and all processes should undergo an external peer review by the auditors. The survey manager should follow procedures for monitoring on an ongoing basis to determine whether the policies and procedures related to the standards are suitably designed and effectively applied. These internal control processes must be guided by the following standards:
- Control environment of the Audit Process

- Risk assessment of timing and resources

- Control activities of the Audit Process

- Communication between the Audit Team and Senior Staff

- Monitoring of all Audit Processes

These standards are applied to all seven phases of a survey's lifecycle. Each of these standards are then broken into individual component parts that follow the general workflow schedule of a U.S. federal government survey from its development to its final dissemination.

As a part of the audit, the Audit Team should review the peer review process to assess their analysis approach and results. Usually this peer review is conducted by the survey manager along with other subject matter, methodological, and statistical experts. All peer reviews should be documented and supporting documentation supplied to the auditors so that the Audit Team can verify that the review was conducted as described. Comprehensive documentation is required to ensure that the audit's quality control system conforms to professional standards in the conduct of its work. Another primary goal of this quality assurance process is to provide each program area a good idea of what quality assurance we are expecting in their program prior to the auditors beginning the external audit. Since many different staff work to complete this checklist or internal audit, we strongly suggest that each staff sign their initials so that it is clear whom to contact should questions arise.

\section{Quality control integration evaluation}

In this section we describe the embedding of our quality control integration evaluation. This quality control provides explicit measurement of quality in processes involved in data collection, data production and data dissemination. The primary goal for inserting this quality control check is to assure our data products are of the highest quality possible. When quality managers recognized repeated failure results from multiple levels of audits, they identified more systematic problems within the enterprise systems and thus, a quality control impetus for change. Below are some common systemic issues the Quality Audit Staff identified:

- Lack of a nonresponse bias analysis

- Improper document storage

- Inadequate planning for the release of data products 
The first common issue resulted from a lack of knowing how and when survey programs should conduct a nonresponse bias analysis. The second common issue resulted from a lack of proper survey documentation and inappropriate or missing documentation. The third common issue related to a lack of procedures for unanticipated data release; that is, how to notify data users that previously released data had been corrected. Another issue the Quality Audit Staff identified is that documentation existed, but it did not match what was actually done. To mitigate these issues in the future, it is important to insert quality control procedures into the tracking systems. These self-documents will detect and provide evidence if the program complies with an OMB standard. When problems are identified, the Quality Assurance Team should report these issues to upper management immediately.

In general, audit results are often looked at from a perspective of "how can we improve?", "what do we need to do to fix the problem?" and "when do we implement it after the problem is fixed?" These common questions need to be addressed between the survey manager and the Quality Audit Staff and the survey manager must identify ways to correct them.

\subsection{Develop a quality control process}

"When implementing any quality control systems, we are not only confronted with business and technology challenges, but most important of all we are faced with managing change" (Lyons E., President of Lyons Information Systems, Inc.) A quality control process must be developed and implemented to track all activities in a survey's life cycle before getting to the point of assessing potential issues and to propose potential solutions. For instance, to address the question of how and when survey programs should conduct a nonresponse bias analysis, the quality control procedure should clearly state that nonresponse bias analyses must be conducted when total response rate is lower than 70 percent. Furthermore, the program managers must appropriately measure, adjust for, and analyze unit and item nonresponse to assess their effect on data quality and to inform data users. In conjunction with the actions that the program manager taken, the Quality Audit Staff must develop a clear understanding of what is needed in the system. This includes a concise understanding of what quality assurance information should be collected, what reports are to be produced, and where the sources of data reside that will be used in the system rather than just checking to see if there is evidence that programs are documented. This requirement will serve as a first filter to pass an initial quality audit.

\subsection{Engage frequent quality control reviews}

Engagement of quality control reviews allow us to evaluate the judgements made by the Quality Audit Staff and their conclusions reached in formulating in the auditor's report. Since budgets are limited, the team must consider the most efficient ways to employ our resources while maintaining our schedules for publication data. This process requires the cooperation of staff in the Economic Directorate so that the reviews can be performed on a timely basis and can also allow the team to respond to findings appropriately. These are some examples of the self-document quality control reviews and quality requirements that must be developed into the tracking systems.

\begin{tabular}{ll}
\hline Procedures & Requirements \\
\hline $\begin{array}{l}\text { Training of staff involved } \\
\text { in data collection and data }\end{array}$ & Objective evidence: \\
analysis: & \\
a. Monitoring and evaluat- & All metrics regarding data col- \\
ing the quality of data col- & $\begin{array}{l}\text { lection and follow-up are tracked } \\
\text { on a daily and monthly basis }\end{array}$ \\
lection operations & $\begin{array}{l}\text { Edits must be monitored on a } \\
\text { routine basis to ensure that edits } \\
\text { b. Monitoring the quality orforming as expected and }\end{array}$ \\
edits & $\begin{array}{l}\text { are providing quality output. } \\
\text { Imputation response rates are } \\
\text { tracked and analyzed over time } \\
\text { in an appropriate manner and } \\
\text { clearly documented in the track- } \\
\text { ing report }\end{array}$ \\
\hline
\end{tabular}

\subsection{Quality assessment and completeness}

Quality assessment checks must be performed to ensure the accuracy and completeness of all survey activities. An important requirement is to develop a clear understanding of what is needed in our program. If necessary, the Quality Audit Team will analyze reports and increase the level of communication of performance. All audit-related documents are needed so that processes can be replicated and evaluated. All documents produced must be retained, consistent with applicable policies of the Census Bureau and data user agreements. These documents must be available to Economic Directorate employees who need them to carry out their work. For instance, if any issues are found during the editing process (this is not necessar- 


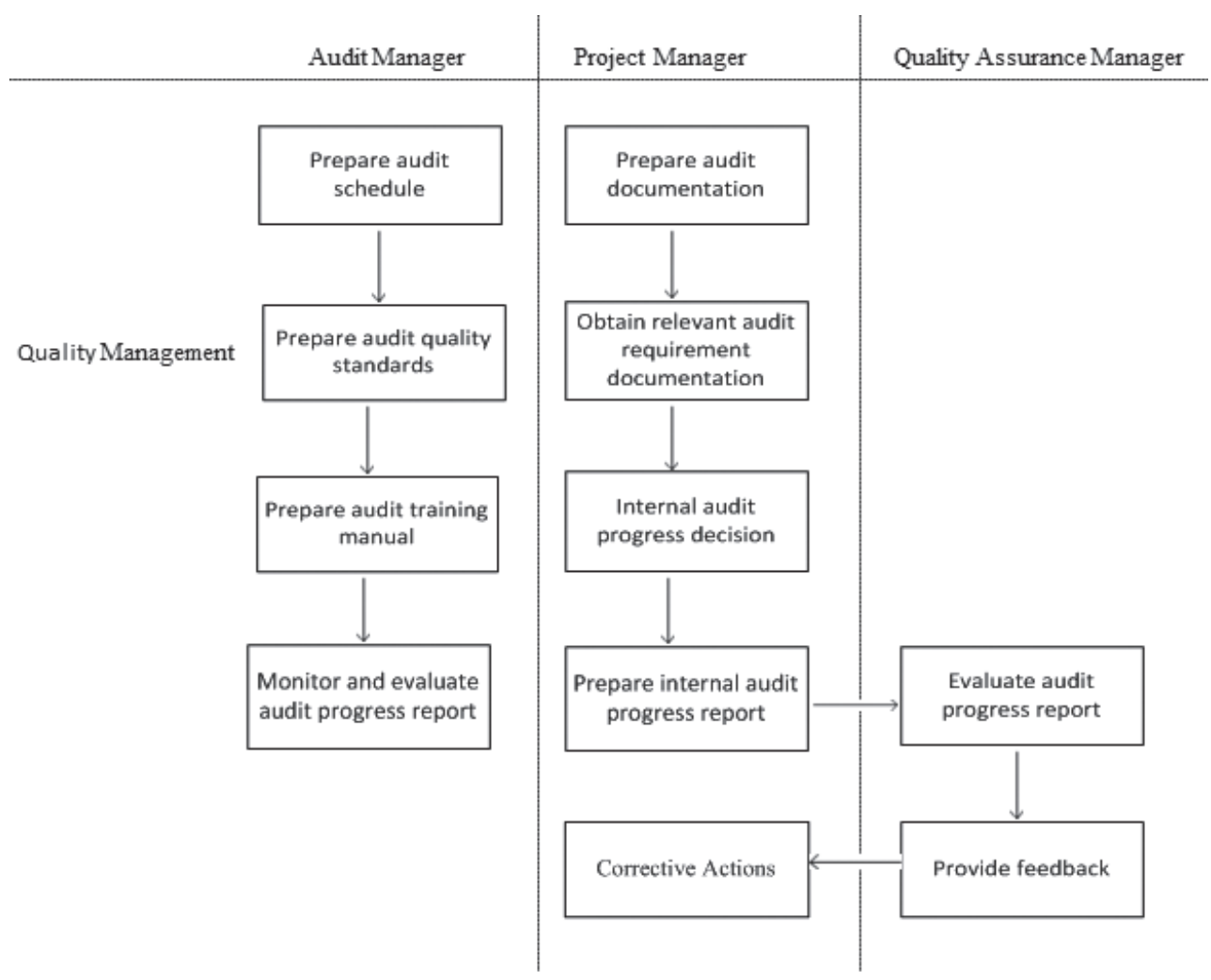

Fig. 2. Quality control activity diagram of audit process evaluation.

ily the issues found during the auditing process), analysts and managers should document these issues and clearly provide corrective actions. Since the audit is performed by individuals with different levels of experience, the survey manager must determine if erroneous dissemination was caused by systematic errors or by the survey analysts. To assess these issues, the survey manager will analyze the quality control results of processing systems (e.g., error rates from clerical coding staff) and develop improvements to systems (e.g., improving clerical coding tools or improving training for clerks). The survey manger must also provide periodic summaries of quality control results and error rate measures.

\section{The future of the economic directorate's quality audit program}

Waning response rates, gaps in the economic data needed to make policy decisions, and a desire to produce more relevant economic measures have led the Economic Directorate to look at the use of third party data sources and modeling as a way to improve eco- nomic statistics while reducing respondent burden. As the Census Bureau makes decisions on how the quality of such measures will be examined and on how third-party data should be evaluated for quality, the Economic Directorate will be examining its procedures for compliance to new standards. The Directorate is also evaluating the current editing processes as well as the edits that will be used for Big Data to determine whether our data are being over-edited. Resources can be directed towards other parts of the Survey Life Cycle and away from over-review of the data.

During the time that the quality audits are redirected, the Quality Audit Staff will also train new managers on the standards and on the reasons for the standards. Taking a kaizen approach, the Quality Audit Staff will aim to build better decision makers and better problem solvers through their understanding of the standards. The Quality Audit Staff will also encourage managers and staff to look at current practices to see if key performance indicators have resulted in wrong behaviors that are counterproductive to continuous improvement and excellence. Staff will be encouraged to examine the current culture to examine if it is focused on "making things better" or "making people better" [1]. This 
training will continue in the future so that the human component of our processes will also be focused on quality. In the future when questions arise about the quality of the methodology or processes, managers will have a better foundation for making quality-based decisions.

After the hiatus from the current quality audit program and after new systems and procedures have been improved using what we know to date, a new audit program will delve deeper into the processes. Whereas the current program checks to see if there is evidence that survey processes are documented, the new audit program will look at the documentation and processes to determine the accuracy of the documentation. Self-documenting systems can be built that will ensure that the documentation matches what systems and programs are doing. The new audit program will also focus on the statistical soundness of the methodology that is being used. The new program will determine if the documentation is detailed enough to yield a repeatable process. Would new staff be able to follow the documentation and reproduce the estimates within an acceptable tolerance? In summary, as a part of the proposed improvements, a new mechanism for auditing the processes, procedures, and methods will be put in place and training of new managers, as well as employees, will continue.

\section{Conflict of interest}

Any views expressed are those of the author and not necessarily those of the U.S. Census Bureau.

\section{References}

[1] J. Miller, M. Wroblewski and J. Villafuerte, Creating a Kaizen Culture: Align the Organization, Achieve Breakthrough Results, and Sustain the Gains. Chapter 1, 2014.

[2] Office of Management and Budget Standards and Guidelines for Statistical Surveys. (September 2006) https://www.white house.gov/sites/default/files/omb/assets/omb/inforeg/statpolicy /standards_stat_surveys.pdf.

[3] International Auditing and Assurance Standards Board, A Framework for Audit Quality (May 15, 2013).

[4] U.S. Census Bureau Statistical Quality Standards (Revised July 2013). http://www.census.gov/content/dam/Census/about/ about-the-bureau/policies_and_notices/quality/statisticalquality-standards/Quality_Standards.pdf.

[5] J. Fowler and S. Klement, Using Aggregate Quality Audit Findings as the Nexus for Organizational Change, 2010.

[6] S. Wagner and M. Meisinger, Integrating a Model of Analytical Quality Assurance into the V-Modell XT.

[7] E. Lyons, Assessing the Assessors: Evaluating Quality Audit Tracking Software Systems. 